\title{
Dual system for management of natural and anthropogenic emergencies and its training
}

\author{
G. A. Sevilla, A. D. Acquesta \& G. M. Giráldez \\ Instituto de Investigaciones Cientificas y Técnicas de las Fuerzas \\ Armadas, República Argentina
}

\begin{abstract}
Natural or anthropogenic emergencies change the way in which a community works, generating crisis situations and preventing the community from recovering from the impact by itself. These emergencies may destroy power, communication and drinkable water supply networks, routes of access, engineering works, crops, factories, homes and human lives.

Frequently institutions are forced to respond to those situations with information that is not complete, up-to-date or reliable. Dissimilar organizations must work jointly and use the material and human assets available in an effective way.

Decisions that have a direct effect on the lives of hundreds or even thousands of people are made within short periods of time and with a high degree of uncertainty.

This work aims at approaching the problem in an integral way, presenting the design of an informatics system that enables crisis management as well as training for the members of the organizations involved in the response to a natural or anthropogenic emergency.

We propose an Internet-supported multi-user system with tools that enable the registering, follow-up and monitoring for support requirements, access to an integrated database of available material and human assets, communication and coordination resources among participants, phenomenon mathematic model support for analysis and prognosis, and an external simulation engine to build and carry out emergency management exercises.

Keywords: crisis management, training system, emergency management, natural phenomena, simulation model, risk analysis, risk assessment, risk management, information and communication issues, population protection, control management and protection.
\end{abstract}




\section{Motivation}

During the emergency management, several factors appear that might complicate to a greater or lesser extent the tasks of those who must respond to the crisis. The following factors may be considered:

\section{Characteristic of the nature of the phenomenon:}

- The phenomenon status can't be known either in real time or to its full extent.

- There is uncertainty about the phenomenon evolution and it is difficult to get accurate forecasts.

\section{Characteristic of the organization:}

- $\quad$ The necessary procedures are not up-to-date, familiar or even present.

- $\quad$ There are difficulties to do emergency management exercises.

- The organization is not familiar enough with the information about its own assets - existence, location, status, personnel in charge, involvement and availability.

- The functions and responsibilities of individuals during an emergency are not properly determined.

\section{Characteristic of the communication among organizations:}

- Information about the assets of other organizations involved - existence, location, status, personnel in charge, involvement and availability - is unknown.

- Information gathered by other organizations is unknown.

- The communication among organizations is poor, infrequent or nonexistent.

- Plans and actions by other institutions are unknown.

\section{Characteristic of the information quality:}

- Different organizations have different information about the same facts.

- $\quad$ Each organization tends to trust in its own means of perception exclusively.

- The means to perceive and capture information are insufficient.

- The information access policies are inadequate.

The aim of the design presented in this work is to approach the difficulties caused by these factors.

\section{Management system}

The management system must give support during the emergency response phase in an integral way. This can be achieved by unifying the information necessary to make decisions, establishing communication among the groups of different organizations that must interact, and offering joint logistic planning and coordination tools.

\subsection{Description}

The management system is an IT system that collects all information available for an organization and sets tools to manage, profit from and share that information with other institutions. 
The system has a client-server multi-user architecture, uses an Internet connection and is designed to be failure resistant by means of a server ring with data redundancy and load distribution.

By means of a proper profile setting policy and adequate information access permissions, the system offers the users the relevant tools to carry out the tasks pertaining their functions. The operations respective to each specific user responsibility are grouped in separate components.

Different types of information from different sources are integrated with a geographic information system in Situation databases without preventing the access to the data being updated.

\subsection{Users}

The information goes through the system in the shape of reports (GIS, Message, Form, News, Request entry, Update, etc.).

Each organization has a data base named Situation database, accessible to all its groups. Every time this database is modified, all users connected to it through the Internet will see the information update. Every organization can share and publish information. Note that, for the system purposes, the organization only operates through its groups. The only IT element that belongs to the organization as a whole is its Situation database.

Published information may be viewed by all organizations but can only be modified by the source one. Shared information can be edited by more than one organization. Only the source organization can access the information which has not been published or shared.

The system includes the set of organizations from which several Internetconnected groups with specific responsibilities depend. Seen as a whole, this system should be able to receive: messages, request entries, updates (about assets, requests, phenomena, etc.), forms, forecasts, alerts, and news. It should be able to send: messages, commands, plans, decisions, forecasts, alerts.

Each group of an organization can only access to the Situation database information related to its responsibilities. These groups may have their own database for assessment purposes that enables to run phenomenon evolution models to analyze possible developments of the events.

Each system user belongs to a particular group, each one of them with very different responsibilities, such as: entering support requirements, entering human or material assets, planning involvements, updating requirement status, updating requirement status, writing reports, sending messages, generating forecasts, broadcasting alerts, issuing orders, monitoring phenomena, assessing risks, registering news, generating situation letters, assessing statistics, etc.

The tasks carried out by a group during the emergency management can be distributed among several users with the same profile, which is determined by the group responsibilities. Although all users of the same group shares the same profile, each user may be devoted to different activities or the same activity applied to different regions. 
The profiles gather several interface modules called components, highly independent from each other, that enable the group to carry out the activities comprising its responsibility.

The following list proposes a number of components and their offered operations:

Message service: Viewing, sending, receiving, searching and printing messages. News: Viewing, writing, importing, publishing, searching and printing news.

GIS: Viewing, modifying, importing, exporting, searching, monitoring, sending and receiving geographical table and document information.

Chat: Viewing, sending and receiving instant messages.

Assets: Adding, removing, modifying, searching and consulting information related to the status, location and involvement of the available assets to respond to the emergency (e.g. consumables, personnel, vehicles, facilities, etc.).

Requests: Entering the request, involve assets, issuing orders, monitoring progress, and entering updates.

Users: Creating, deleting, modifying and consulting information about users.

Groups: Creating, deleting, modifying and consulting information about organization groups.

Statistics: Creating, viewing and printing management statistics.

A permission policy should be offered to limit the viewing and modifying of information only to groups who have that responsibility. This allows the different decision-making levels to access more or less summarized information and therefore avoid data excess. On the other hand, this policy allows keeping the layer modification access restricted.

The system should trigger visual or sound alarms when a certain piece of information requires the user's attention. The use of permissions ensures that the information received by a user will be relevant to his/her responsibilities.

\subsection{Information organization}

Each organization has its own Situation database that consists of the information available to that organization at the beginning of the emergency management. Part of this information might be modified throughout the emergency by means of updates from relevant groups. This data base could include the following initial information: bridges, settlement points, international borders, internal borders, road network, river network, train network, police stations, firefighters, hospitals, schools, evacuation centers, storage centers, contour lines, risk maps, optic and radar satellite images (in normal status); assets: vehicles, equipment, personnel, consumables, mattresses, medicines, blankets, drinking water, requests; phenomenon information: flooded region, land saturation, digital elevation model, optic and radar satellite images, earthquakes, faults, tectonic plates, volcanoes, spills; communications: messages, news, geographical reports, forms.

After determining the validity, opportunity and completeness of the new pieces of information, the profiles with data base modification capacities update the Situation database. 
Dynamic information is a set of tabular data and layers that describe the current status of the situation. This information remains valid because of its capacity of change and describes reality aspects evolving throughout the whole management process. This information is used to learn about the current status of the emergency and make decisions accordingly. Dynamic information can be modified, shared and published. By sharing dynamic information, other organizations will have the capacity (and the responsibility) of modifying it; the system is in charge of synchronizing it. By publishing dynamic information, other organizations may see it but can't modify it.

Static information is formed by the set of received and accumulated reports which modified the situation. Since they have a specific date and time of issuing, they are just event notifications, that can't be modified and may quickly lose its validity. When these reports are applied to the Situation database, they change the dynamic information status. Static information can be generated, sent and received. When static information is sent, a report is distributed among the relevant groups and organizations. Static information will not change the dynamic information of the Situation database until it is applied to it.

Only published or shared dynamic information uses automatic synchronization tools to provide other organizations with continuous access to up-to-date information.

\subsection{Architecture}

The system is developed following a client-server approach. Permanent workstations, like those for monitoring or risk assessment, have a dedicated client application connected to the organization server. Temporary workstations, like update entry or order reception stations, can access the system through a web browser. The system uses a dynamic number of servers. There has to be communication among all servers to maintain information redundancy, synchronize shared or published information layers, ensure system operational capacity in the event of failures and spread processing load among them during the emergency response.

\subsection{Cooperation and coordination}

Communications go among participants in the shape of reports. This way the system can save a log with every communication, message, decision and update, which will enable a future analysis of the efficiency of procedures or a study of the relevance of modifying them.

Messages are viewed by integrating a standard e-mail server to the system. This way the user can have their mailbox and other components necessary to comply with their functions during management process in a single tool.

Users in charge of media exploitation have a tool to publish articles from different sources (press, radio, TV, digital media, etc.) relevant to the emergency management in the system. These articles will be displayed to the users by means of the news component, which has a digital newspaper structure and allows the user to access news from previous days and search articles by both title and body. 
The system accepts both vector and raster geographic information in standard formats. When published geographical information is modified, every user with permission to view that information will receive those updates and the GIS component will activate an alarm and show the updated information.

The forms are pieces of text information entered in a specific format to be able to be automatically processed and then generate statistics that evolve with the reception of each new form. For instance, if a daily report detailing the number of evacuated people classified by gender and age is requested, the user in charge of filling it in will enter the data in a numeric format, and once the form sent, the system will be able to add them automatically and process them accordingly.

The request entries are records of necessities communicated to the operators (by phone, radio, etc.) whose responsibility is to enter them to the system. Once the request has been entered, an alarm will be triggered and the users in charge of analyzing the request will be able to devote assets to act to it. In order to do so, the users will need to have access to the assets future engagements and know their availability status. Since assets information includes information about their personnel in charge, the user will be able to send the orders directly to the individuals in charge of each asset for them to act to the request.

\subsection{Phenomena analysis}

The participating groups may have the responsibility of maintaining and running mathematic models to analyze the possible evolution of a phenomenon in the near future.

A model is an algorithm that, from the current data base status, processes the received stimuli to generate the following status of that database. These models represent the relation between a phenomenon and certain elements common to every natural or anthropogenic emergency, such as evacuation, rescue or medical assistance requests; damages and operability of facilities and equipments; necessity of mattresses, blankets, medicines, food and drinking water; injured, missing and deceased people; security problems in health centers; evacuation; storage, etc. The models may be used to study the more likely evolution of the situation during emergency management.

The result of this process gives the opportunity to prepare a response for future events. Since experts in different areas have the knowledge about these models, the system offers a generic language that enables the description of a large number of mathematic models that can be loaded and installed in a model server and used taking the Situation data base as its basis. As this data base consists of the best current information the organization has about the emergency, the system should offer the groups the possibility of working on a copy of these data which can be modified without affecting the information accessible by the other groups. This new database owned exclusively by the group is called Study, and all its layers may be copied from the Situation database and modified freely.

Models may be used for the generation of forecasts and alert bulletins to be sent later to other parts of the management process as reports. 
The better the aspects involved in a natural or anthropogenic emergency are represented, the more useful the forecasts will be.

\section{Training system}

An important part of our proposal is to think of the training system as the management system immersed in a simulated environment. This way, the management system is also used during training, offering the following advantages over having two independent systems:

- The user is familiar with the interface before the emergency management process.

- Upgrades in the management system are immediately available for training.

- The management system is constantly tested.

- The training cost and effort are lower.

- The system maintenance cost and effort are lower.

- The simulation tools and the management system are highly independent.

- In the training system, the trainees do not perceive the directors controlling the exercise, bringing a complete immersion in the simulation.

In the training configuration, the simulation server and the exercise director application are added to the servers from each organization and the group applications.

The simulation server is in charge of supporting the event tree, triggering the events and its associated reports, enabling the directors to control and monitor the execution of the exercise and applying changes to the Reality data base, which describes constantly the current status of the simulation.

As in the management system, the profiles enable the customization of the interfaces for each user - director, trainee, observer, etc.

In the director profile, the following components are included:

Exercise management: to create exercises, prepare them and control their execution.

Events: to schedule the event tree ramifications, events, reports and changes.

Tables: to manage users, groups and profiles.

\subsection{Users}

During the exercise, the trainees represent the groups that take part in a crisis management, preferably with the same responsibilities they would have during a real crisis. The directors control the delivery of reports to the trainees, the simulated time passage and the phenomena evolution.

The director interface must be able to send reports impersonating groups interacting with the trainees during the exercise. The trainees do not perceive any difference between information from other trainees or from the direction, since all data are treated in the same manner.

\subsection{Data organization}

The Reality database simulates an emergency evolution in a dynamic way. It is the origin of the information in the reports that are sent to each group and update 
the Situation database of its organization. Note that, since these reports are not necessarily complete, truthful nor timely, the Situation database of each organization may show different information, as it is the case in a real emergency. Trainees should use the management system capacities to view, modify, share and publish the information of their Situation data bases in order to form collectively a depiction of the situation as close to the simulated reality as possible.

In a real emergency management, each organization has means, external or of their own, to perceive changes and enter updates. In training, these means of perception - inspectors, reconnaissance flights, damage assessment equipments, victims, involved personnel, etc. - are simulated through IT agents called perceptors. The behavior of the perceptor depends on its type and the orders received from the trainees. For instance, an optical satellite perceptor could add cloud clusters and flooded areas to a clear satellite image using the Reality data base data for a time $\mathrm{T}$, every $\mathrm{N}$ hours, and send the report to the group that requested those images with a delay of $\mathrm{D}$ hours. On the other hand, the inspector perceptors could send updates about the area to which they were assigned with no delay, but with greater spatial limitations. This way, the system enables the training of operators to make decisions with uncertainty, since each organization has a subjective view of the situation.

\subsection{Architecture}

To carry out training exercises for emergency operators, the management system receives reports from a simulation server. This way, the operation of the management system is not altered by being immersed in a training context and can offer its capacities - geographical representation, document organization, information exchange, logistic planning - in a genuine manner. Director applications offer the possibility of creating and modifying the events of the event tree, impersonating the groups not represented by trainees, monitoring communications and modifying the Reality data base directly or by means of stimuli sent to the model server to keep or change the course of events. Many directors may work on the exercise data at the same time, so an adequate concurrence policy must be implemented.

\subsection{Exercise direction}

For the preparation of an exercise, an educational goal at which the direction aims should be stated first. Next, a list of participating groups should be included, together with the designation of a trainee or a director as the impersonator for each group. The system maintains an event tree, that is to say, a tree-shaped event structure with multiple courses of events to richly describe the foreseeable development for the exercise. The tree events include changes for the Reality database, reports to be sent to groups and stimuli for mathematic models. The better the aspects involved in a natural or anthropogenic emergency are represented, the more realistic the exercises will be. The evolution of a phenomenon can also be described by simply scheduling consecutive events that change the phenomenon state. 
Exercises already prepared may be totally or partially reused, progressively simplifying the process of preparation of new exercises.

The director interface controls the simulated time passage, enabling time jumps, changes in the time passage rate, and stopping and resuming time passage.

While the exercise is being executed, events can still be added, deleted, modified, activated and deactivated to adjust the development to the educational goals of each exercise session.

\section{Conclusions}

The dual system for management and training reduces the negative effects of the factors exposed before in the following way:

Characteristic of the nature of the phenomenon:

- The use of the system will derive in a greater knowledge of the phenomenon status, since information acquired by any group can be shared immediately, and changes in a greater spatial extension can be known.

- The system increases the possibilities for forecast generation by means of the execution of mathematic models.

- Forecasts published in the system are seen by the participants automatically, without having to make additional operations or queries.

- Characteristic of the organization:

- The system enables the organization to have a greater access to information - existence, location, status, personnel in charge, involvement and availability - about its own assets.

- The system enables a greater access to information - existence, location, status, personnel in charge, involvement and availability - about the assets of the other organizations.

- The practice of crisis management exercises aids in the writing, testing and updating of procedures for real emergencies.

- The use of the system reduces to a great extent the difficulties to do emergency management exercises.

- The system will be a progressive aid in the determination of functions and responsibilities of each user during an emergency, and the statement of adequate information access policies.

- Characteristic of the communication among organizations:

- The system enables the access to information obtained and published by other organizations.

- The system improves the communication among organizations.

- The system provides information about the plans and actions by other organizations to coordinate joint operations.

- Characteristic of the information quality:

- The system enables the organizations to access more information that is more up-to-date.

- The system enables its users to receive updates captured by means of perception owned by different organizations. 
- The system increases the information sources available for each organization.

- The system can maintain adequate information access policies by means of profiles and permissions associated to each group.

Dealing with an emergency situation poses a great challenge, and its resolution involves coordination, rationality, assertiveness, efficiency and speed in the decision-making process. To increase the likelihood of success, it is extremely important that an adequate training program be offered that is close to reality, adaptable to multiple organizations and different scales, thus giving the opportunity to teach both the tactical-operational and the strategic levels and without removing the personnel from their workplace. This enormous responsibility is significantly relieved when a dual system with the characteristics mentioned above is available. By the use of the capacities of the proposed design, we are convinced that we will have more and better opportunities to survive a crisis satisfactorily, with the consequent benefits for the society.

\section{Acknowledgements}

Translation credits: Romina L. Ferraro and Patricia N. Rosenberg.

\section{References}

[1] Borkulo, E. van, Scholten, H.J., Zlatanova, S. and den Brink, A van, 2005. Decision making in response and relief phases, Geo-information for disaster management.

[2] Bowers III, F. A. and Prochnow, D. L., 2003. JTLS-JCATS Federation Support for Emergency Response Training, Proceedings of the 2003 Winter Simulation Conference.

[3] Mendonça, D. and Fiedrich, F. 2004. "Training for Improvisation in Emergency Management: Opportunities and Limits for Information Technology." International Journal of Emergency Management.

[4] Turoff, M., Chumer, M., Van de Walle, B. and Yao X., 2004. "The Design of a Dynamic Emergency Response Management Information System (DERMIS)", The Journal of Information Technology Theory and Application (JITTA). 